\title{
LE POIDS DU MOT GENRE DANS LES REVENDICATIONS DE «L'ÉCRITURE INCLUSIVE »
}

\author{
Philippe SELOSSE \\ Université Lyon 2 - UMR 5317 IHRIM
}

\begin{abstract}
For gender studies, concord with masculine gender reflects sexism. This paper defines the signification of the French word genre as a formal category, with a marking function in sentences, which has nothing to do with sex. Grammatical gender in language is arbitrary and artificial, as many historical examples can demonstrate, except among the prototypical group of animated and sexed beings. As the nomenclature of genders " masculin vs féminin » (masculine vs feminine gender) is the consequence of this prototypical categorization, it should be given up in linguistics, on behalf of the use of gender conceived as purely grammatical, opposing « marked vs unmarked » gender.
\end{abstract}

Pour Alicja, en souvenir amical de nos rencontres scientifiques placées sous le signe de la nomenclature et de la taxinomie...

\section{INTRODUCTION}

En sciences sociales, les gender studies ont posé la distinction terminologique et conceptuelle du genre (gender) comme 'norme sociale hiérarchisante reposant sur des rôles sociaux de masculinité / féminité' et du sexe comme 'réalité biologique reposant sur des attributs biologiques (mâle / femelle)'. En 2005, la Commission générale de terminologie et de néologie récusait cet anglicisme, au motif que le mot genre empiétait indûment sur le terrain sémantique du paradigme du mot sexe (sexiste, sexuel...). Mais le cœur des «études genre » (sic) étant de déconnecter sexe biologique et genre social, la préconisation d'en rester au mot sexe ne pouvait être satisfaisante : l'usage militant a alors tout emporté et le mot genre est passé 
- dans les médias, les formations universitaires, les travaux académiques et une partie des textes administratifs. Qu'en est-il de la justesse de cet emploi, eu égard au schéma conceptuel du mot et à son noyau sémantique rémanent (Honeste, 2011) ? En quoi participe-t-il d'une ancienne mais tenace et nuisible confusion des niveaux d'approche?

\section{1. À L'ORIGINE DU GENRE : MISE AU POINT SÉMANTIQUE}

Genre est issu du latin genus 'origine, extraction, naissance', d'où l'emploi 'réunion d'êtres ayant une origine commune [famille, maison, race, nation]' qui se retrouve dans genus humanum 'genre humain' ; en dérive l'emploi taxinomique du nom genus pour signifier une 'classe', un 'groupement'. Le nom latin genus est issu du verbe gignere 'engendrer', qui se rattache à une racine indo-européenne 'gne 'engendrer'. On retrouve ces traits sémantiques dans les très nombreux mots de la famille : générer et engendrer ('donner naissance'), gendre ('celui qui engendre'); gens ('ceux qui se caractérisent par une origine commune'), génocide ('destruction d'un genre, i. e. d'une communauté ethnique partageant une origine commune'), gentil ('propre à la gens, i. e. à la race', d'où 'de bonne race, noble' puis 'généreux') ou dégénérer ('perdre les qualités de sa race, de son genre') ; génie (initialement, 'divinité qui préside à la naissance de quelqu'un') ; génital ('qui sert à engendrer, donner naissance') et géniteur ('celui qui engendre, qui donne naissance'); indigène ('originaire d'un pays') ; engin (du latin ingenium 'caractère inné', d'où dérivent en français les emplois métonymiques 'intelligence, inspiration, talent innés et personnels', puis 'machine, outil [procédant de cette intelligence]') et ingénieux, ingénieur, etc. Le trait sémantique commun, constant, est celui d'origine, donnée (engendrer) ou obtenue (naître) selon le contexte et les affixes lexicaux. À partir du concept de point commun donnant naissance à une classe, d'ensemble de traits à l'origine d'un groupement homogène, la langue a par métonymie désigné la catégorie résultante elle-même - le genre. C'est ainsi que le mot genre est utilisé en français où " l'idée générale de "groupement, catégorie" domine l'ensemble des emplois au cours de l'histoire » $(D H L F)$, pour la catégorie naturelle (genre d'animal ou de plante), philosophique (genre d'objets ou d'êtres), grammaticale, etc. - tout comme le nom genus était ainsi majoritairement utilisé en latin.

Le mot sexe, lui, est issu du latin sexus, dont l'étymon est incertain, le plus probable étant le latin secare 'couper, diviser, partager'; il renvoie aux caractères, organes, fonctions... qui partagent un genre en mâles et en femelles. En termes taxinomiques aristotéliciens, genre est la catégorie et sexe, la différence : du point de vue lexicologique et philosophique, il y a donc différence de contenu et de niveau entre genre et sexe. 


\section{LA CATÉgORIE DU GENRE EN LANGUE}

\subsection{DÉFINITION LINGUISTIQUE}

Pour les grammairiens et linguistes, le mot genre est conçu comme

une catégorie grammaticale reposant sur la répartition des noms dans des classes nominales, en fonction d'un certain nombre de propriétés formelles qui se manifestent par la référence pronominale, par l'accord de l'adjectif (ou du verbe) et par des affixes nominaux (préfixes, suffixes ou désinences casuelles), un seul de ces critères étant suffisant. Ainsi, d'après ces trois critères, on définit en français deux classes, les masculins et les féminins : Le prince est mort ; il était encore un enfant s'oppose à La princesse est morte ; elle était encore une enfant, par la référence pronominale (il / elle), par l'accord (mort / morte, un enfant / une enfant) et par les affixes nominaux (- / -esse) [...]. (DLSL, art. « Genre»)

La catégorie du genre se manifeste comme propriété formelle, à l'instar du nombre : c'est un balisage commode qui permet l'identification des syntagmes et leur repérage ou leur suivi, par l'anaphore, dans la phrase et/ou d'une phrase à l'autre : rien de sexuel dans cette économie linguistique.

Reste toutefois la question de l'interprétation sémantique de ces genres en soi et de leur attribution à tel ou tel mot. $\mathrm{Si}$ « la majorité des langues du monde (57\%) ignorent complètement le genre, la plupart des langues à genre distinguent 2 à 3 classes » (Martinie, Patri, 2014) et

dans les descriptions linguistiques des langues connaissant deux classes, un des genres est pris comme base du système (cas non-marqué), les autres genres étant décrits relativement à lui (cas marqués) : en français, le féminin est généralement décrit par une variation morphologique du masculin pris comme base (le féminin maîtresse est décrit par l'adjonction de l'affixe -esse au masculin maître). [...] À cette catégorisation relevant de propriétés formelles (genre grammatical) est associée le plus souvent une catégorisation sémantique (genre naturel) relevant d'une représentation des objets du monde par leurs propriétés spécifiques. Les classifications les plus constamment associées sont :

a) l'opposition entre les personnes et les objets (animés et non-animés), les non-animés étant neutres relativement à la distinction de genre masculin / féminin $[\ldots]$;

b) l'opposition de sexe à l'intérieur des animés entre mâle et femelle. (DLSL, art. « Genre ») 
Les linguistes distinguent alors deux mécanismes de catégorisation en général :

(1) La motivation sémantique : l'attribution d'un genre à un nom résulte de ce que les propriétés des référents sont sémantiquement convergentes.

(2) La motivation morphologique : l'attribution d'un genre à un nom résulte de ce que les propriétés des lexèmes sont morphologiquement convergentes. (Martinie, Patri, 2014)

Dans le cas (1), le fait que les référents soient sentis comme formant une classe cohérente leur fait assigner un genre commun : lièvre et verrat sont des mâles et en tant que tels forment un genre commun, qui se marque conséquemment par un genre grammatical commun A; hase et truie sont des femelles et en tant que telles forment un autre genre commun, qui se marque conséquemment par un autre genre grammatical commun B. Dans le cas (2), le fait qu'un mot possède tel affixe lui confère automatiquement tel genre, commun à tous ceux possédant cet affixe; ainsi les noms dérivés d'un verbe avec le suffixe -ure sont féminins, tandis que ceux dérivés par le suffixe -ment sont masculins : déchirer $>$ une déchirure, un déchirement; racler > une raclure, un raclement.

Doit-on considérer que les genres A et B ont été affectés dans le cas (2) sur le modèle du cas (1) et qu'ils sont motivés sémantiquement ? Dans cette hypothèse, les noms en -ment seraient au masculin parce qu'exprimant un processus mâle, dynamique, opératif ; les noms en -ure seraient au féminin parce que renvoyant à un processus femelle, statique, résultatif. Ou doit-on considérer que les genres A et $\mathrm{B}$ ont été affectés dans le cas (1) parce qu'on en disposait déjà pour marquer les inanimés du cas (2) ? Dans ce cas, les genres seraient pleinement arbitraires : de même que les genres auraient été attribués aléatoirement pour raclure et raclement, ils l'auraient été pour lièvre et hase - l'essentiel étant de disposer dans la phrase de marques de différenciation des mots par le genre et de marques identiques pour les membres d'une catégorie, quand elle existe (telle la catégorie sexuelle). On ne saurait trancher dans l'état des connaissances historiques mais on veillera à éviter :

1. de réduire le mécanisme morphologique du genre à quelque chose de purement morphologique, tout au moins dans la langue première (en indo-européen), où on peut douter que les genres aient été distribués arbitrairement, sans aucune signification élémentaire ;

2. de considérer, au terme d'évolutions historiques complexes, que le mécanisme sémantique d'attribution, perceptible pour les animés sexués, est à étendre à tous les autres mots. C'est la dérive classique des partisans de «l'écriture inclusive » qui en conduit certains à s'indigner que la pièce 
la plus forte soit de moindre valeur que le billet le plus petit - vision réductrice et partiale qui néglige qu'un billet est une coupure et qu'une pièce est un sou...

\subsection{Arbitraire dU GENRE : L'EXEMPLE DU FRANÇAIS}

Le genre des noms résulte, en français comme dans d'autres langues, de facteurs multiples (phonétiques, sociaux, culturels, etc.), arbitraires au sens où ils n'ont rien à voir avec la motivation sémantique. L'exemple des doublets lexicaux est assez remarquable à cet égard du caractère aléatoire tout au long de l'histoire. Trois cas de figure se rencontrent, selon que le genre de l'étymon latin...

1) se retrouve dans les deux formes françaises : articulus (n. m) $>$ orteil (n. m.) et article (n. m.) ; flos, floris (n. f.) > fleur (n. f.) et flore (n. f.) ;

2) se retrouve dans une seule des formes françaises, généralement la savante, la forme populaire étant de genre différent : musculus (n. m.) $>$ moule (n. f.) et muscle (n. m.) ; potio (n. m.) > poison (n. m.) et potion (n. f.). Cf. le triplet polypus (n. m.) > poulpe (n. m.), pieuvre (n. f.) et polype (n. m.), où les formes populaires sont elles-mêmes de genres variés pour désigner le même type d'animal ;

3) ne se retrouve dans aucune des deux formes du doublet français (faute de neutre dans cette langue), mais avec des résultats variés : ministerium (n. n.) > métier (n. m.) et ministère (n. m.) ; vitrum (n. n.) > verre (n. m.) et vitre (n. f.).

Tout est possible, sans prédictibilité certaine. Et quand il y a prédictibilité dans d'autres cas, on ne saurait rien en déduire : si les noms neutres n'ont pas donné lieu à des noms neutres en français, la répartition s'est faite, en général, entre noms latins neutres au singulier aboutissant à des noms français masculins (p. ex. granum $>$ grain, folium $>$ a. f. fueil), et noms latins neutres au pluriel aboutissant à des noms français féminins (p. ex. grana $>$ graine, folia $>$ feuille). On pourrait objecter le sentiment récurrent du locuteur qui, soucieux inconsciemment d'un système linguistique régulier, affectera à une forme terminée en - $e$ un genre « féminin ». C'est cette recherche de système dans la langue qui expliquerait, entre autres, que des mots originellement masculins ou neutres en latin soient devenus et restés féminins en français (p. ex. comète, étude, idole, n. f. en français $v s$ en latin cometes, n. m. et studia et idolum, n. n.), malgré les tentatives des humanistes de rétablir le genre étymologique - et qu'à l'inverse, des mots à finale consonantique, dont le genre était féminin en latin, soient devenus masculins (p. ex. art, poison) ; et c'est ce même sentiment de système qui expliquerait 
l'erreur récurrente sur cernes, effluves et tentacules, noms masculins souvent donnés pour féminins par les locuteurs. Mais les exemples ci-dessus démentent déjà cela (cf. les masc. article, muscle, poulpe, ministère ou verre, tout comme le simple nom... homme) ; par ailleurs, erreur, humeur, odeur..., sont bien passés au genre féminin, contrairement au latin d'origine, introduisant de la disparate dans les noms suffixés en -eur, généralement masculins (cf. honneur, labeur...); et on connaît les cas où, bien au contraire, le genre féminin d'une forme à finale en -e paraît incongru-ainsi, parler d'une espace en typographie donne souvent lieu à une résistance des locuteurs ignorants de cette terminologie. Toute analyse objective de l'histoire des mots doit conduire à bannir la tentation de surinterprétation en féminin pour les finales en - $e$, ou en masculin «dominateur» dans d'autres cas, pour tout ce qui relève des noms renvoyant à de l'inanimé et à une bonne partie de l'animé (cf. poulpe et pieuvre...) $)^{1}$.

\subsection{Mauvais genre}

Tous les exemples qui précèdent n'ont pas été donnés pour le plaisir de l'anecdote. En effet, nombre de personnes favorables à « l'écriture inclusive» s'attaquent au genre masculin des noms comme si ce genre grammatical signifiait le sexe mâle. Au vu de débats sur les réseaux sociaux et revues militants (Aeschlimann, 2017), "l'écriture inclusive » n'est pas loin de préconiser de réserver, pour un signifié semblable, les emplois des mots profession, idée, assemblée, maison..., pour les femmes, et ceux de métier, concept, parlement, domicile..., pour les hommes. Par ailleurs, lorsque les partisans de " l'écriture inclusive » (MESF, 2017) prônent l'accord de proximité dans l'exemple Les coteaux et montagnes voisine $\underline{\text { s, }}$ pour refuser la « domination » du masculin, ils reproduisent le présupposé que le genre grammatical, masculin ou féminin, est une manifestation sexuée. Or, dès qu'on a pris conscience de l'arbitraire du genre grammatical du nom, on doit avoir simultanément à l'esprit que l'accord des mots se rapportant au nom, quel que soit le genre de celui-ci, procède de cet arbitraire : si coteau et montagne sont respectivement masculin et féminin arbitrairement, accorder au masculin ou au féminin porte la même arbitrarité pour les mots accordés avec le nom et ne signifie rien de sexuellement mâle ou femelle. La stigmatisation d'une langue sexiste renvoie les tenants de "l'écriture inclusive » à une parfaite ignorance

${ }^{1}$ La place manque pour développer cette restriction mais on rappellera que même dans le cas des animés, le sexe n'est pas prédictible à partir du genre grammatical : voir les cas connus de $\boldsymbol{l a}$ grenouille ou $\boldsymbol{d} \boldsymbol{u}$ crapaud, tous deux avec représentants mâle et femelle, du mannequin femme et de la sentinelle homme, etc. 
diachronique et synchronique du système linguistique, du sens et de la fonction de la catégorie de genre. La juste critique devrait viser le discours machiste, qui sexualise le genre grammatical masculin, et la revendication féministe, qui sexualise le genre grammatical féminin, et les renvoyer dos à dos.

\section{LA CONFUSION DES GENRES}

\subsection{Catégorisation ET NOMENClature PROTOTYPIQUES}

Il est un mécanisme de catégorisation bien connu en nomenclature qui pourrait aider à comprendre les sources de la confusion et qui abonde en langue : celui de la dénomination d'un niveau super-ordonné à partir du nom du niveau sousordonné, conçu comme prototypique. La langue courante connaît cela en toutes catégories grammaticales : a) le nom huile (< lat. oleum, latinisation du gr. elaion, forme neutre d'elaia 'olive') signifie spécifiquement le 'liquide gras de l'olive' mais sert de prototype pour désigner génériquement la catégorie des liquides gras végétaux (huile de noix, de sésame...) et même animaux (huile de foie de morue $^{2}$; b) l'adjectif de couleur orange, conversion du nom orange, renvoie à un fruit spécifique jugé représentatif pour s'appliquer génériquement à tout corps présentant ladite couleur, y compris d'autres fruits ; c) le verbe saupoudrer, litt. 'poudrer de sel (sau-)', est un acte spécifique étendu génériquement à tout processus consistant à couvrir d'une matière pulvérulente, d'où saulpoudrer de sel dès $1370(D M F)$, d'épices dès 1555 (Selosse, 2014), de sucre / de farine en français moderne, etc. De même, en nomenclature scientifique, c'est un nom de genre de plante (p. ex. Rosa) qui sert de type pour forger le nom de la famille (les Rosacées). En linguistique, la catégorie des déterminants possessifs est dénommée relativement à la situation prototypique de possession ( $C$ 'est mon livre de linguistique, pas touche!), alors que les contextes d'emplois peuvent être bien différents (Ce n'est pas « mon » cours de linguistique! s'écrie l'étudiant de lettres, qui n'envisagerait même pas de revendiquer la propriété d'une telle discipline).

Dans tous ces exemples, on peut toujours constater et contester le fait arbitraire ou conventionnel de recourir à tel élément pour dénommer toute la catégorie, qu'il s'agisse d'un état donné de culture où le sel prévaut, d'une vision européanocentrée faisant de la rose la fleur par excellence, etc. Mais il serait absurde de critiquer le fait que ce soit le sel et non le sucre, la rose et non la potentille, qui aient prévalu dans le choix de la dénomination.

${ }^{2}$ Sur la déconnexion immédiate de l'expérience particulière pour signifier une expérience conceptuelle, voir Honeste (2015). 


\subsection{USAGE ET RISQUES D'UNE NOMENCLATURE PROTOTYPIQUE}

En langue courante, la catégorisation prototypique, et la nomenclature consécutive, ne sont plus problématiques : la motivation du mot se perd, de sorte qu'il devient possible de parler d'une huile d'olive ou d'une carotte orange, sans qu'on y voie pléonasme ou monstruosité botanique. En langue de spécialité, en revanche, on préfère éviter l'inconvénient des dénominations partiales, le mode de dénomination prototypique risquant d'emporter avec lui une vision faussée, à l'aune de l'évolution des connaissances. C'est pourquoi, dans l'exemple des « possessifs », certains linguistes (Wilmet, 1998) privilégient la dénomination de "déterminants personnels », du fait de la commutation sémantique avec un SP intégrant le pronom personnel de référence $($ sa voiture $=$ « la voiture de lui $»)-$ la relation personnelle pouvant être ou non de propriété.

Pour en revenir à notre confusion genre / sexe, elle ressortit au procédé exemplifié ci-dessus. La saillance du sous-groupe des animés sexués, jugé prototypique, conduit à dénommer l'ensemble de la catégorie grammaticale du genre à partir des caractéristiques associées à ce sous-groupe, caractéristiques sexuelles dont procèdent les dénominations génériques de "masculin » et " féminin ». Il en ressort que, pour le genre, ce n'est pas le sexe masculin qui est à la source du problème catégoriel et nomenclatural, c'est la prééminence prototypique de la distinction sexuelle, masculin et féminin, qui brouille la notion depuis des lustres.

Certes, comme on l'a vu, on peut s'accommoder sans risque d'une appellation prototypique impropre et l'académicienne Danielle Sallenave rappelle par expérience qu' " enseigner l'accord au masculin, genre grammatical [souligné à l'oral], ne conduit pas les petits garçons à se frapper le torse » (France-Inter, 24 novembre 2017). Mais la formulation machiste du «masculin qui l'emporte sur le féminin » pouvant prêter à... confusion, le plus simple pourrait être de supprimer tout simplement les dénominations de « masculin » et « féminin » qui conduisent à repenser le système de codage générique en système de discrimination sexuée ${ }^{3}$. Et on pourrait alors, du point de vue nomenclatural, leur substituer d'autres noms, en revenant par exemple à la terminologie neutre, descriptive et didactique de la linguistique : respectivement, genre non marqué vs genre marqué.

${ }^{3}$ Il serait tout aussi bon que grammaires et ouvrages de linguistique, dans leur exemplification du genre, cessent de recourir à des noms sexués (tels les prince et princesse dans la citation de la section 2.1). 


\section{Conclusion}

Que la nomenclature du genre grammatical récupère le couple prototypique masculin / féminin a pu encourager des grammairiens (Vaugelas, Beauzée) à plaquer sur le genre un discours sexué / sexiste. Mais si le fondement des « études genre » est de déconnecter socialement genre et sexe, qu'elles en fassent de même linguistiquement et empêchent à la source la projection d'une idée de sexe sur une catégorie générique qui en est vierge ! À vouloir défendre le genre grammatical en tant que féminin, les partisans de «l'écriture inclusive » ne contribuent qu'à entériner l'identification genre-sexe, à substituer à un placage idéologique un autre qui n'est guère plus justifié - somme toute, à biaiser l'étude neutre d'une réalité objective et à fausser la compréhension du système linguistique.

\section{Références bibliographiques}

\section{Ouvrages et articles}

AESCHLIMANN, Richard J. (2017), "Une genre de nouvelle discrimination chromatique », GLAD! Revue sur le langage, le genre, les sexualités, $\mathrm{n}^{\circ}$ 3, www.revue-glad.org/842.

BONNARD, Henri (1989), « Le Genre », Grand Larousse de la Langue Française, Paris, Larousse, pp. 2201-2211.

COMMISSION GÉNÉRALE DE TERMINOLOGIE ET DE NÉOLOGIE(2005), «Recommandation sur les équivalents français du mot "gender" », Journal Officiel du 22 juillet 2005.

HONESTE, Marie Luce (2011), « Le phénomène de rémanence et ses conséquences en sémantique lexicale à travers l'histoire du mot opinion », Le Français préclassique, n 13, pp. 91-113.

HONESTE, Marie Luce (2015), «Une approche expérientielle de la sémantique lexicale », Intellectica, $n^{\circ}$ 64/2, pp. 87-112.

MARTINIE, Bruno, PATRI, Sylvain (2014), Linguistique générale. La catégorisation nominale (genre et classes), Licence 3 de Sciences du Langage, Université Lyon 2.

SELOSSE, Philippe (2014), «Pierre Belon, La Nature et diversité des poissons (1555), Philippe Glardon éd., Genève, Droz, 2011 » [compte rendu], Le Français préclassique, $\mathrm{n}^{\circ} 16$, pp. 217-242.

WILMET, Marc (1998), Grammaire critique du français, Paris, Hachette / Duculot.

\section{Dictionnaires}

DHLF : Dictionnaire historique de la langue française (1992), réd. A. Rey et al., Paris, Société du Nouveau Robert. 
DLSL : Dictionnaire de linguistique et des sciences du langage (1994), réd. J. Dubois et al., Paris, Larousse.

DMF : Dictionnaire du Moyen Français (1330-1500) (version 2015, actualisée au $1^{\text {er }}$ février 2016), ATILF, ATILF-CNRS-Université de Lorraine, http://www.atilf.fr/dmf/.

\section{Sitographie}

MESF : Manifeste « Nous n'enseignerons plus que "le masculin l'emporte sur le féminin" » (2017), www.slate.fr/story/153492/manifeste-professeurs-professeures-enseignerons-plus-masculinemporte-sur-le-feminin (dernière consultation : le 12.01.2018). 